\title{
ARTICLE
}

\section{Longitudinal follow-up of dome-shaped macula}

\author{
Tomas R. Burke ${ }^{1,2} \cdot$ Angela Ding $\mathrm{Wu}^{1} \cdot$ Yuening Shen ${ }^{1} \cdot$ Ranjan Rajendram ${ }^{1}$
}

Received: 7 August 2019 / Revised: 8 January 2020 / Accepted: 13 January 2020 / Published online: 22 January 2020

(c) The Author(s), under exclusive licence to The Royal College of Ophthalmologists 2020

\begin{abstract}
Background/objectives To determine if the presence of sub-retinal fluid (SRF) was associated with reduced vision in domeshaped macula (DSM), and to assess its effect and response to treatment during follow-up.

Methods Patients were identified retrospectively. Baseline and follow-up data were recorded. The diagnosis of DSM, and presence or absence of SRF and intra-retinal fluid (IRF) was confirmed using Spectral Domain-Optical Coherence Tomography (SD-OCT). Decisions to treat oedema were based on clinician preference.

Results 193 eyes of 106 patients (71 female) were confirmed to have DSM. Overall mean duration of follow-up for this cohort was 3.5 years. Mean BRVA for all eyes at baseline was 0.38 (range: -0.20 to 'light perception'). A significant difference was noted in mean baseline BRVA between those eyes with SRF compared with those without SRF at baseline ( 0.48 vs. $0.31, p<0.001)$. Intra-retinal fluid moderately correlated with poorer baseline BRVA $(r=0.31, p<0.003)$. No significant change in BRVA was noted during follow-up. No significant effect of treatment on BRVA was observed.

Conclusions The presence of SRF at baseline was associated with poorer vision. Vision appears to remain stable irrespective of the presence or absence of SRF at baseline. The treatments administered in this cohort did not affect final vision or SRF.
\end{abstract}

\section{Introduction}

Dome-shaped macula (DSM) is an inward bulge of the retinal pigment epithelium (RPE) within the chorioretinal concavity of the posterior pole based on optical coherence tomography (OCT) [1]. Initially described in myopic eyes with staphyloma, DSM has been reported in emmetropes, hypermetropes as well as those with a range of other diagnoses [2]. Suggested mechanisms for the development of DSM include localised hypotony and vitreo-macular traction [3], focal choroidal thickening [1, 4, 5], as well as localised scleral thickening [6]. Central macular sub-retinal fluid (SRF) is present in a proportion of patients with DSM,

This study was carried out at Moorfields Eye Hospital, London, UK.

This paper was presented at EURETINA 2018 in Vienna, Austria on Sept $21^{\text {st }}, 2018$.

Tomas R. Burke

tomasburke@nhs.net

1 Department of Ophthalmology, Moorfields Eye Hospital, London, UK

2 Present address: Department of Ophthalmology, Bristol Eye Hospital, Bristol, UK but there is conflicting data as to its negative effect on visual acuity (VA) [5, 7-14]. Furthermore, there are no clearly effective treatments for SRF in eyes with DSM. In this report, we present the baseline and longitudinal data in a large cohort of patients with DSM. VA data were compared in those eyes with and without oedema. The effect of administered treatments was also assessed.

\section{Materials (subjects) and methods}

Patients with DSM were identified retrospectively from an electronic patient record system (OpenEyes ${ }^{\mathrm{TM}}$ www. openeyes.org.uk/) using the search term "dome" to search through all patient correspondences that contained the keyword at Moorfields Eye Hospital, London, UK. Identified correspondences, and the associated medical records and imaging data, were scrutinised. Non-DSM cases were excluded. Baseline data were collected on all patients and, where available, final follow-up data were also collected. These time-points were each defined as that relevant clinic visit where OCT imaging and best-recorded visual acuity (BRVA) data were available, and an interval up to a maximum of 2 months between BRVA and OCT imaging was considered as 1 visit. Clinical, including BRVA and refraction data, as well as demographic data were collected 
on all patients. Data relating to ocular co-morbidities and administered treatments were also recorded. Approval for this study was granted by the Audit Department of Moorfields Eye Hospital (\#140).

\section{Cross-sectional and en-face imaging}

Imaging on all patients had been performed using Spectraldomain (SD)-OCT acquired with the Topcon 2000 (Topcon Corp, Tokyo, Japan) and/or the Heidelberg Spectralis (Heidelberg Engineering, Heidelberg, Germany). The diagnosis of DSM was confirmed by the lead author (TB) using macular scans acquired in at least one axis, with DSM described as an inward bulge of the RPE line of at least 50 um, as previously described [10]. Depending on clinical practice, different patterns of scans had been performed in the horizontal, vertical and/or diagonal axes.

The presence of intra-retinal fluid (IRF) and SRF was recorded. To facilitate identification of the sclero-choroidal junction, choroidal thickness measurements were only performed using enhanced-depth imaging (EDI, Heidelberg) scans. As the horizontal scan was the most commonly performed EDI scan in this cohort, these scans were used for choroidal measurements to maximise consistency of results. All measurements were performed in the 1:1 um mode using the proprietary in-built HEYEX software (Heidelberg Engineering, Heidelberg, Germany) using the calliper tool. Thickness of the choroid was measured perpendicularly from the outer border of the RPE:Bruch's membrane (BM) complex to the sclero-choroidal junction in the centre of the fovea (Fig. 1). In a small number of cases the suprachoroidal space was visualised, and this was included in the total thickness of the choroid. A similar method was used to calculate the thickness of central macular SRF from the inner border of RPE:BM complex to the tips of the outer segments of the displaced photoreceptors.

Fundus autofluorescence (FAF), fundus fluorescein angiography and indocyanine green angiography were acquired in selected cases. These en-face images were

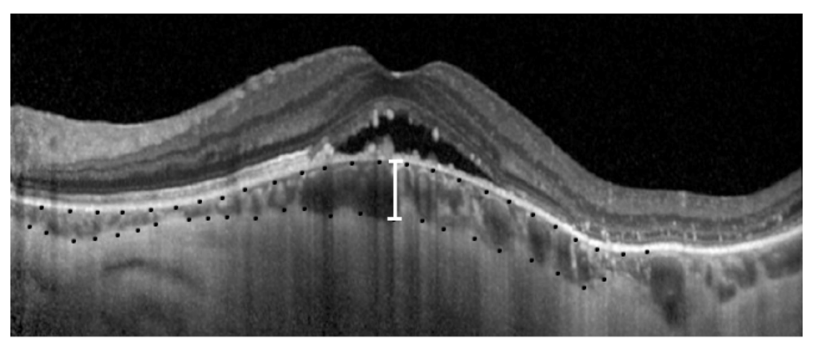

Fig. 1 Example of dome-shaped macula with sub-retinal fluid. Thickness of the choroid was measured from the outer border of the RPE:Bruch's membrane (BM) complex, visualised as a hyperreflective band, to the sclero-choroidal junction in the centre of the fovea. acquired on either the Heidelberg Retina Angiograph (Heidelberg Engineering, Heidelberg, Germany) and /or Topcon 2000 (Topcon Corp, Tokyo, Japan). For those eyes with available data, they were separated into three groups based on the appearance of the foveal FAF signal: normal, speckled hyperAF and hypoAF consistent with fovea involving atrophy.

\section{Statistical analysis}

For statistical analysis, BRVA was converted to LogMar. Eye(s) with perception of light (PL) vision were excluded. Analysis was carried out in Microsoft Excel, using the XLSTAT plug-in (Addinsoft, France 2018). Correlation between BRVA for both eyes from each patient, where available, was calculated to determine if both eyes from each individual could be treated individually for analysis. Mann-Whitney $U$ test was used to compare BRVA for baseline and follow-up visits, while a Wilcoxon signed-rank was used for paired data. In selected analysis, where the relevant data were normal distributed, $t$ test was used. Fisher's exact test was applied for paired categorical data. A $p$ value of $<0.05$ was considered statistically significant. Kurtosis within a range of \pm 2 were acceptable to prove normal distribution for BRVA. Univariate logistics regression was used to identify those variables with significant correlations. Where relevant, these included age at baseline, mean spherical equivalent refractive error, duration of follow-up, presence or absence of SRF at baseline and/or follow-up, presence or absence of IRF at baseline and/or follow-up, FAF appearance (see above) and administered treatments. Those variables found to have a significant association were then assessed by multivariate logistics regression. Repeatability of choroidal thickness measurements $(\mu \mathrm{m})$ was based on those values obtained at baseline visits by two observers (TB, AD) and was assessed using the Coefficient of repeatability (CoR) which is directly related to the $95 \%$ limits of agreement proposed by Bland and Altman [15]. Where large differences between individual measurements were noted, these were re-measured with open adjudication.

\section{Results}

Demographic and clinical information for this cohort is summarised in Table 1. In total 193 eyes of 106 patients (71 female) were confirmed to have DSM following review of the available SD-OCT imaging, with associated BRVA data, to permit analysis. The mean age at presentation was 53.3 years (rang: 7.2-79.0). Refraction data were available for 130 eyes in 65 patients. In 120 of 130 of eyes (92\%) the refraction was myopic. 68 eyes were highly myopic $(\leq-6)$. Mean spherical equivalent refractive error for all patients 
Table 1 Summary of patient characteristics at baseline.

\begin{tabular}{ll}
\hline Age $(\mathrm{yrs})$ & $53.3( \pm 15.5)$ \\
Gender & Female $71 /$ male 35 \\
Spherical equivalent (dioptres) & $-7.8( \pm 6)$ \\
Eyes/patients & $193 / 106$
\end{tabular}

Age and refraction data are presented as mean \pm standard deviation.

was -7.8 dioptres sphere (range: +1.75 to -24.50 ). Missing refraction data was an issue in 63 eyes, while 7 eyes were emmetropic or low-hypermetropic. Note that Baseline and follow-up BRVA data were not normally distributed. The median duration of follow-up for this cohort was 3.3 years (range: $0.1-9.5$ ). Only 16 eyes had follow-up of $<1$ year.

In 16 patients, DSM was present in only one eye and so only data from the affected eye was included in the analysis. In three additional cases data were only available for one eye. One eye had PL vision and was excluded from statistical analysis.

In terms of co-morbidities, these were in keeping with those that would be expected in a population of predominantly highly myopic patients and are summarised in Table 2.

Interestingly, five patients (10 eyes) also had a diagnosis of Keratoconus $(\mathrm{KCN})$. Four patients had already been diagnosed, while one was diagnosed during follow-up. One of these patients also had a diagnosis of Oculocutaenous albinism. Another five patients were younger than 20 years of age (range 7.2-18.4 years). One patient each had Bornholm's disease and another Interphotoreceptor matrix proteoglycan 2 (IMPG2) retinopathy. A selection of these patients have previously been reported in another publication from our institution [2].

\section{Visual acuity (baseline)}

Mean BRVA for all eyes at baseline was 0.38 (range: -0.20 to PL). As correlation in baseline LogMar BRVA between eyes was low $(r=0.27, p=0.012)$, data from patients with information available for both eyes was included and analysed independently. Baseline BRVA for right and left eyes were similar (0.40 (range: -0.20 to PL) and 0.37 (range: -0.20 to 3 ), respectively). 79 eyes (40.7\%) had SRF at baseline, while $113(59.3 \%)$ had no SRF at baseline. A significant difference was noted in mean baseline BRVA between those eyes with SRF compared with those without $\mathrm{SRF}$ at baseline $(0.48$ vs. $0.31, p<0.001)$. This difference was also present when all eyes with co-morbidities that could affect vision were also excluded from analysis $(0.28$ vs. $0.4, p=0.003$ ).

Of the 107 eyes with FAF data available at baseline and abnormalities identified (atrophy or speckled hyperAF),
Table 2 Summary of co-morbidities/ocular complications.

\begin{tabular}{ll}
\hline Diagnosis & \# Affected patients \\
\hline Choroidal neovascular membrane & 34 \\
Pseudophakia & 17 \\
Epiretinal membrane/lamellar hole/full- & 12 \\
thickness macular hole/ vitreo-macular traction & \\
Glaucoma/ocular hypertension & 9 \\
Amblyopia & 8 \\
Corneal dystrophy (keratoconus) & $7(5)$ \\
Retinal detachment & 7 \\
Cataract & 7 \\
Laser refractive procedure & 6 \\
Punctate inner choroidopathy/presumed ocular & 5 \\
histoplasmosis & \\
Retinopexy & 4 \\
Retinitis pigmentosa/cone-rod dystrophy/retinal & 3 \\
dystrophy & \\
Anterior uveitis & 2 \\
Penetrating keratoplasty/collage cross linking & 2 \\
Bornholm disease & 1 \\
Alligallie Syndrome & 1 \\
Diabetic macular oedema & 1 \\
Pattern dystrophy & 1 \\
Aphakia & 1 \\
Non-arteritic anterior ischaemic optic & 1 \\
neuropathy & 1 \\
Choroidal naevus & 1 \\
Oculo-cutaneous albinism & \\
\hline & \\
\hline
\end{tabular}

lower mean BRVA was present compared with those eyes with normal FAF ( 0.47 vs. $0.11, p<0.0001)$. On multivariate logistics regression only SRF, IRF and FAF appearance had significant correlation with baseline BRVA, however, their effects were low but for IRF that had a moderate effect $(r=0.31, p<0.003)$.

\section{Visual acuity (follow-up)}

Final follow-up data were available on 151 eyes. Of the 68 eyes with SRF at baseline, there was no significant difference in BRVA between baseline and follow-up ( 0.47 vs. $0.46, p=0.48)$. Forty-four $(65 \%)$ of these eyes had persistence of SRF at final follow-up, however, again no significant change in mean BRVA was noted (0.51 vs. 0.51, $p=0.86$ ). Twenty-four eyes (35\%) with SRF at baseline, but no SRF at follow-up, also did not reveal a significant change in vision $(0.41$ vs. $0.37, p=0.29)$. These data are summarised in Table 3. Of those 83 eyes with no SRF at baseline, no significant change was detected in BRVA at follow-up, including sub-group analysis of those 74 (89\%) eyes that remained SRF free, and the nine $(11 \%)$ eyes that 
Table 3 Final follow-up data.

Wilcoxon signedrank test

\begin{tabular}{|c|c|c|c|}
\hline \multirow{5}{*}{$\begin{array}{l}\text { No SRF at } \\
\text { baseline }\end{array}$} & No. of eyes & 83 & \\
\hline & Baseline BRVA & 0.41 & \\
\hline & Final follow-up BRVA & 0.37 & $p=0.29$ \\
\hline & SRF at final follow-up & $9(11 \%)$ & \\
\hline & $\begin{array}{l}\text { No SRF at final } \\
\text { follow-up }\end{array}$ & $74(89 \%)$ & \\
\hline \multirow{5}{*}{$\begin{array}{l}\text { SRF at } \\
\text { baseline }\end{array}$} & No. of eyes & 68 & \\
\hline & Baseline BRVA & 0.47 & \\
\hline & Final follow-up BRVA & 0.46 & $p=0.48$ \\
\hline & SRF at final follow-up & $44(65 \%)$ & \\
\hline & $\begin{array}{l}\text { No SRF at final } \\
\text { follow-up }\end{array}$ & $24(35 \%)$ & \\
\hline
\end{tabular}

had SRF at final follow-up. Importantly, multivariate regression analysis did not identify a significant effect of duration of follow-up on final BRVA or on the presence of SRF at final review. The median durations of follow-up in eyes with and without SRF at baseline was 3.5 and 2.9 years, respectively. However, this difference was not found to be significant $(p=0.459)$. The ranges between groups were similar between those with and without SRF at baseline, with minimum follow-up of 0.1 and 0.2 years, respectively, and both with maximum follow-up of 9.5 years.

A sub-group of 49 eyes had complete FAF data available at baseline and follow-up. Controlling for the powerful effect of baseline BRVA, multivariate regression analysis identified that only IRF at baseline and duration of followup had a correlation with follow-up BRVA, but these were low ( $r=0.03$ and $r=0.2$, respectively). Importantly, correlation between baseline and follow-up FAF appearance was high $(r=0.97, p<0.001)$, with only five eyes showing progression of FAF changes over follow-up within this subgroup (one from normal to speckled hyperAF, four from speckled hyperAF to atrophy).

\section{Effect of treatment}

We were interested to determine the effect of treatment(s) on the presence of SRF and BRVA at follow-up. Treatments administered were heterogenous. The treatment decisions were 'observation', anti-VEGF therapies, photodynamic therapy (PDT), focal laser and Epleronone. A subgroup of 63 eyes with SRF at baseline had available followup and treatment data. Of the 41 eyes with SRF at followup, $18(44 \%)$ had received treatment while $23(56 \%)$ had not. Of the 22 eyes with no SRF at follow-up, $13(59 \%)$ had received treatment, while $9(41 \%)$ had not been treated.
However, no significant effect of treatment on the presence of follow-up SRF was detected $(p=0.3)$.

The most common management decision was observation (32 eyes). Anti-VEGF therapies were administered in 20 eyes, Eplerenone in six eyes, while PDT and focal laser were administered in four and three cases, respectively. Some eyes received more than one treatment. There was no significant difference in the BRVA at baseline in those eyes that received treatment compared with those that did not ( 0.52 vs. $0.45, p=0.19)$. There was also no significant difference in BRVA from baseline to follow-up in either group. Based on angiography, seven of those eyes treated with anti-VEGF therapy had definite CNVM, and again no difference between baseline and follow-up vision was noted. However, poorer vision was present at baseline in this sub-group of eyes with CNVM compared with those without CNVM (0.64 vs. $0.46, p<0.05)$. This difference was not maintained at follow-up ( 0.49 vs. $0.46, p=0.46)$. No significant difference was noted in final BRVA based on whether treatment was given for baseline SRF or not $(0.51$ vs. $0.42, p=0.44)$.

\section{Choroidal thickness and presence of SRF}

EDI data were available for 57 eyes. Choroidal thickness measurements at baseline were obtained using the horizontal EDI scans. While the mean value of sub-foveal choroidal thickness was greater in those without SRF at baseline compared with those with SRF $(206 \mu \mathrm{m}$ vs. $239 \mu \mathrm{m}$, respectively), the difference was not statistically significant $(p=0.38)$. CoR was $14.5 \%$ between the two observers for choroidal thickness measurements. In a sub-set of 11 eyes with central macular SRF at baseline and/or follow-up and available EDI scans, it was noted that the thickness of SRF ranged from $0 \mu \mathrm{m}$ to $250 \mu \mathrm{m}$ across follow-up with a mean range of $115.6 \mu \mathrm{m}$ for individual patients.

We aimed to identify if the peak of the bulge occurred in the sub-foveal region. In 18 eyes the "choroidal peak" was decentred temporally $(505 \mu \mathrm{m}-2650 \mu \mathrm{m})$ from central fovea. In 13 eyes the peak was decentred nasally (54-2445 $\mu \mathrm{m})$, excluding those where the peak appeared within the peripapillary region. In only one eye was the peak directly in the central fovea, while in the remained the peaks were either multiple or too diffuse to clearly identify.

\section{Discussion}

Longitudinal data in patients with DSM has been reported $[8-12,15]$. The greatest mean duration of follow-up in these cohorts was 37.9 months (maximum duration within that cohort was 111 months) [12]. As with our cohort, the majority of previous reports have found no significant 
change in VA during follow-up. It was interesting, however, that in many previous reports no statistically significant difference in vision was reported between those eyes with and without SRF at baseline [5, 9-14]. In only one study a significant difference has been detected between those eyes with and without SRF at baseline [7]. In our report we also found this difference to be statistically significant. As with other reports, the presence of RPE abnormalities was associated with poorer visual acuities in our cohort [1, 7], though we found limited progression of these abnormalities during follow-up. A feature of the macular oedema that has not been interrogated previously in DSM was the effect on BRVA of IRF. In our cohort, there was a moderate correlation between poorer baseline BRVA and IRF. This feature may represent a sub-type of DSM patients with a more severe phenotype, or may relate to chronicity of disease as is recognised in central serous retinopathy [16].

In keeping with other reports $[9,12]$, no significant benefit was noted in terms of benefit to VA or a consistent effect on SRF based on treatments administered. Subthreshold laser was not utilised in this study and a positive effect on vision has been reported with its use previously [8].

It has been reported that $\mathrm{CNV}$ is not significantly associated with DSM [17]. Not surprisingly, eyes with CNVM had poorer vision at baseline than those without [17], and this was noted in our cohort also. Reassuringly, the BRVA did not change significantly over the course of follow-up in our cohort of patients with CNVM.

Variations in choroidal thickness across the macula in cases of DSM have been reported [7, 11, 18-20]. Deobhakta et al. identified focal variations in choroidal thickness in a series of patients with SRF [18]. In our cohort, we focused on examining if the peak of the bulge was to be found in the central sub-foveal area, however, it was not in a majority of cases. This is interesting as it supports the observations that it is not solely the sub-foveal location of the bulge of the scleral and choroid that is responsible for DSM, but likely a combination of their combined morphology that contributes to the development of DSM.

An interesting observation identified in this cohort was the presence of $\mathrm{KCN}$ in five patients. The prevalence of $\mathrm{KCN}$ is variable, depending on the methods used for diagnosis and racial mix of study populations. Nonetheless, $\mathrm{KCN}$ is likely much more common than previously suspected, and a recent report has put the estimated prevalence in the general population at 1:375 (265 per 100,000) [21]. The rate observed in this study is much higher, approximate to $1: 21$. Although the cornea and sclera have different optical properties, largely resulting from the collagen fibril orientation, they have similar collagen content [22]. Interestingly, $\mathrm{KCN}$ is not associated with marked axial elongation [23, 24]. Furthermore, it has been reported that choroidal thickness is increased in patients younger than 45 years old with KCN [25]. The observed higher frequency of $\mathrm{KCN}$ in our DSM patients warrants further investigation to determine if there is an association in terms of pathological disease process.

Our cohort of patients was also unique in that five patients were identified at an age-range $<20$ years. The majority of previously reported cases in the literature are of patients more than 20 years of age [1, 2, 5-7, 9, 11-13, 17], so this sub-group are very young to have been diagnosed with DSM. The youngest of our patients was 7 years of age at presentation, the youngest reported age, to our knowledge, of a patient with DSM in the literature. This suggests that DSM should be considered by clinicians assessing reduced vision in the young, particularly in myopic patients.

Limitations of this study include its retrospective nature and the expected associated biases, including the identification and follow-up of those patients with symptoms and/ or more severe phenotype(s). Lack of standardisation of imaging meant that EDI and/or vertical scans were not available in all cases so that there was missing data for certain sub-analyses. Decisions regarding treatments were not standardised and so caution must be exercised in assessing the effect of treatments in our cohort.

In this study, we set out to evaluate a large cohort of patients with DSM and to examine the long-term effect of this diagnosis with/without SRF on VA. It is re-assuring that VA does not change significantly within the observed follow-up period. It is possible that with longer durations of follow-up, a significant change in may be detected. Prospective work is required to identify appropriate treatments for SRF and IRF in DSM, particularly given that our cohort exhibited a significantly poorer BRVA in the presence of SRF.

\section{Summary}

\section{What was known before}

- Debate exists in the current literature about the effect of SRF on vision in DSM.

\section{What this study adds}

- This report adds the evidence that there is a negative effect of SRF on vision in DSM. However, this was not found to be associated with a further deterioration in vision during the course of follow-up. This large cohort identifies an increased prevalence of keratoconus in patients with DSM. DSM is reported in a sub-group of young $(<20$ years old) patients. 


\section{Compliance with ethical standards}

Conflict of interest The authors declare that they have no conflict of interest.

Consent and ethical statement Patients were assessed as part of normal clinical care with data collection for this study carried out as part of a registered audit at Moorfields Eye Hospital (Audit \#140). Therefore, no specific consent was obtained for each patient. All procedures performed in studies involving human participants were in accordance with the ethical standards of the institution and with the 1964 Helsinki declaration and its later amendments.

Publisher's note Springer Nature remains neutral with regard to jurisdictional claims in published maps and institutional affiliations.

\section{References}

1. Gaucher D, Erginay A, Lecleire-Collet A, Haouchine B, Puech M, Cohen SY, et al. Dome-shaped macula in eyes with myopic posterior staphyloma. Am J Ophthalmol. 2008;145:909-14.

2. Errera MH, Michaelides M, Keane PA, Restori M, Paques M, Moore AT, et al. The extended clinical phenotype of dome-shaped macula. Graefes Arch Clin Exp Ophthalmol. 2014;252:499-508.

3. Mehdizadeh M, Nowroozzadeh MH. Dome-shaped macula in eyes with myopic posterior staphyloma. Am J Ophthalmol. 2008;146:478.

4. Chebil A, Ben Achour B, Chaker N, Jedidi L, Mghaieth F, El Matri L. Choroidal thickness assessment with SD-OCT in high myopia with dome-shaped macula. J Fr Ophtalmol. 2014;37:237-41.

5. García-Ben A, Sanchez MJM, Gómez AG, García-Basterra I, García AS, García-Campos JM. Factors associated with serous retinal detachment in highly myopic eyes with vertical ovalshaped dome. Retina. 2019;39:587-93.

6. Imamura Y, Iida T, Maruko I, Zweifel SA, Spaide RF. Enhanced depth imaging optical coherence tomography of the sclera in dome-shaped macula. Am J Ophthalmol. 2011;151:297-302.

7. Caillaux V, Gaucher D, Gualino V, Massin P, Tadayoni R, Gaudric A. Morphologic characterization of dome-shaped macula in myopic eyes with serous macular detachment. Am J Ophthalmol. 2013;156:958-67.e1.

8. Battaglia Parodi M, Iacono P, Bandello F. Subthreshold laser treatment for serous retinal detachment in dome-shaped macula associated with pathologic myopia. Retina. 2018;38:359-63.

9. Lorenzo D, Arias L, Choudhry N, Millan E, Flores I, Rubio MJ, et al. Dome-shaped macula in myopic eyes: twelve-month followup. Retina. 2017;37:680-6.

10. Ellabban AA, Tsujikawa A, Muraoka Y, Yamashiro K, Oishi A, Ooto $\mathrm{S}$, et al. Dome-shaped macular configuration: longitudinal changes in the sclera and choroid by swept-source optical coherence tomography over two years. Am J Ophthalmol. 2014;158:1062-70.
11. Viola F, Dell'Arti L, Benatti E, Invernizzi A, Mapelli C, Ferrari $\mathrm{F}$, et al. Choroidal findings in dome-shaped macula in highly myopic eyes: a longitudinal study. Am J Ophthalmol. 2015;159: 44-52.

12. Soudier G, Gaudric A, Gualino V, Massin P, Nardin M, Tadayoni $\mathrm{R}$, et al. Long-term evolution of dome-shaped macula: increased macular bulge is associated with extended macular atrophy. Retina. 2016;36:944-52.

13. García-Ben A, Garcia-Basterra I, González-Gómez A, BaqueroAranda I, Morillo-Sanchez MJ, Soler-García A, et al. Comparison of long-term clinical evolution in highly myopic eyes with vertical oval-shaped dome with or without untreated serous retinal detachment. Br J Ophthalmol. 2019;103:385-9.

14. Pilotto E, Guidolin F, Parravano M, Viola F, De Geronimo D, Convento E, et al. Morphofunctional evaluation in dome-shaped macula: a microperimetry and optical coherence tomography study. Retina. 2018;38:922-30.

15. Alakeely AG, Alrashaed S. Serous retinal detachment in domeshaped macula with 7 years follow-up. Middle East Afr J Ophthalmol. 2016;23:323-5.

16. Daruich A, Matet A, Dirani A, Bousquet E, Zhao M, Farman N, et al. Central serous chorioretinopathy: recent findings and new physiopathology hypothesis. Prog Retin Eye Res. 2015;48: 82-118.

17. Liang IC, Shimada N, Tanaka Y, Nagaoka N, Moriyama M, Yoshida T, et al. Comparison of clinical features in highly myopic eyes with and without a dome-shaped macula. Ophthalmology. 2015;122:1591-600.

18. Ellabban AA, Tsujikawa A, Matsumoto A, Yamashiro K, Oishi A, Ooto $\mathrm{S}$, et al. Three-dimensional tomographic features of domeshaped macula by swept-source optical coherence tomography. Am J Ophthalmol. 2013;155:320-8.e2.

19. Deobhakta A, Ross AH, Helal J, Maia A, Freund KB. Localized choroidal thickness variation and pigment epithelial detachment in dome-shaped macula with subretinal fluid. Ophthalmic Surg Lasers Imaging Retin. 2015;46:391-2.

20. Ohno-Matsui K, Fang Y, Uramoto K, Shinohara K, Yokoi T, Ishida $\mathrm{T}$, et al. Peri-dome choroidal deepening in highly myopic eyes with dome-shaped maculas. Am J Ophthalmol. 2017;183: 134-40.

21. Godefrooij DA, de Wit GA, Uiterwaal CS, Imhof SM, Wisse RP. Age-specific incidence and prevalence of keratoconus: a nationwide registration study. Am J Ophthalmol. 2017;175:169-72.

22. Meek KM, Fullwood NJ. Corneal and scleral collagens-a microscopist's perspective. Micron. 2001;32:261-72.

23. Schlatter B, Beck M, Frueh BE, Tappeiner C, Zinkernagel M. Evaluation of scleral and corneal thickness in keratoconus patients. J Cataract Refract Surg. 2015;41:1073-80.

24. Lanier JD, Bullington RH, Prager TC. Axial length in keratoconus. Cornea. 1992;11:250-4.

25. Gutierrez-Bonet R, Ruiz-Medrano J, Peña-Garcia P, Catanese M, Sadeghi Y, Hashemi K, et al. Macular choroidal thickening in keratoconus patients: swept-source optical coherence tomography study. Transl Vis Sci Technol. 2018;7:15. 